\title{
Variation of electrophoretic karyotypes in genetically different strains of Saccharomycopsis fibuligera and Yarrowia lipolytica
}

\author{
B. H. Nga, C. W. Yip, S. I. Koh and L. L. Chiu
}

Author for correspondence: B. H. Nga. Tel: +65 772 3282. Fax: +65 7766872.

Department of

Microbiology, National University of Singapore, Lower Kent Ridge Road, Singapore 0511

\begin{abstract}
Saccharomycopsis fibuligera is a dimorphic yeast, which is both saccharolytic and fermentative, that is used in the production of rice wine. It has a predominant diploid phase. When grown on solid agar 5 . fibuligera strains develop different morphological forms. In previous studies, intergeneric hybrids between S. fibuligera and a related yeast, Yarrowia lipolytica, were obtained which were able to utilize starch and tributyrin. A putative haploid mitotic segregant, N14i60 met, was obtained from the intergeneric hybrid between S. fibuligera 193 met and Y. lipolytica A his1. Auxotrophic mutants were readily isolated following UV mutagenesis of N14i60 met. The auxotrophic mutants and $\mathrm{N} 14 \mathrm{i60}$ have a similar morphology. Protoplast fusants were produced between the auxotrophic mutant A6 met lys1 arg1 and Y. lipolytica 21501-4 B lys5 leu2 ade1 xpr2 and between specific pairs of the auxotrophic mutant strains. Differences and similarities in the DNA banding patterns of these genetically different strains of S. fibuligera and Y. lipolytica were demonstrated and it was established that four distinct types consistent with their specific positions in the pedigree chart could be clearly distinguished. Furthermore, by a study of the patterns of hybridization signals for specific genes of $S$. fibuligera for an intergeneric hybrid and its mitotic segregants, genetic segregants with different characteristics were obtained. The segregants from the intergeneric hybrid and the protoplast fusants probably arose by a process of chromosomal assortment at mitosis. The intergeneric hybrid, the protoplast fusants and their mitotic segregants showed a similar karyotype. Together these studies provide an explanation for the basis of phenotypic differences of some of the yeast strains studied.
\end{abstract}

Keywords: Saccharomycopsis fibuligera, Yarrowia lipolytica, electrophoretic karyotypes

\section{INTRODUCTION}

Saccharomycopsis fibuligera was first isolated from chalky bread by Lindner in 1907 and it was then named Endomyces fibuliger Lindner. In 1984, it was re-classified in the genus Saccharomycopsis (Lodder, 1970; Kreger-van Rij, 1984). Strains of $S$. fibuligera have been isolated from ragi tape and macaroni (Barnett et al., 1983). It is a homothallic yeast, with a dominant diploid phase and a brief haploid phase. It is dimorphic, and exhibits both a multicellular mycelial form with branched septate hyphae which contain plasmodesmata, and individual budding cells. $S$.

Abbreviation: PFGE, pulsed-field gel electrophoresis. fibuligera produces hat-shaped ascospores that are formed in spheroidal asci borne usually on lateral branches of the main hyphae.

When cultivated on yeast extract/phosphate/soluble starch agar (YPSS), strains of $S$. fibuligera give white sporulation. On prolonged culture the colonies often produce mitotic sectors. $S$. fibuligera is a protein secretor, and produces and secretes glucoamylase and $\alpha$-amylase. It is a slow sugar-fermenting yeast; sugars such as glucose and maltose, but not galactose and sucrose, are converted to alcohol and carbon dioxide. $S$. fibuligera is also able to hydrolyse cellobiose by its extracellular $\beta$-glucosidase (Machida et al., 1988). These characteristics make it extremely suitable for use in brewing industries to 
Table 1. Descriptions of yeast strains used in this study

\begin{tabular}{|c|c|c|c|}
\hline Category & Strain & Origin & Description of colonies grown on YPSS \\
\hline I & S. fibuligera $\left.\begin{array}{r}2610 \text { met } \\
8014 \text { met }\end{array}\right\}$ & Stock culture & $\begin{array}{l}\text { Convex white colonies with erose margin } \\
\text { Convex white colonies with erose margin }\end{array}$ \\
\hline II & S. fibuligera 193 met & UV-induced mutant of 8014 met & $\begin{array}{l}\text { Pulvinate brownish colonies with erose } \\
\text { margin }\end{array}$ \\
\hline III & S. fibuligera 56 met & Mitotic sector of 193 met & Pulvinate white colonies with erose margin \\
\hline IV & $\begin{array}{l}\text { S. fibuligera N14i60 met } \\
\left.\begin{array}{l}\text { A6 met lys } 1 \text { arg } 1 \\
\text { A18 met ade1 } \\
\text { I12 met bis } 1\end{array}\right\}\end{array}$ & $\begin{array}{l}\text { Mitotic sector of the } \\
\text { intergeneric hybrid } 193 \\
\text { met/1529 A bis } 1 \\
\text { UV-induced mutants of N14i60 } \\
\text { met }\end{array}$ & $\begin{array}{l}\text { Thin, filmy white colonies with less aerial } \\
\text { mycelia than strains of categories I, II and } \\
\text { III }\end{array}$ \\
\hline $\mathrm{V}$ & $\begin{array}{l}\left.\begin{array}{l}\text { S. fibuligera } / Y \text {. lipolytica intergeneric } \\
\text { hybrid } \\
\text { A6/21501-4B } \\
\text { A18/21501-4B }\end{array}\right\}\end{array}$ & Protoplast fusion & Colonies with the appearance of 193 met \\
\hline VI & 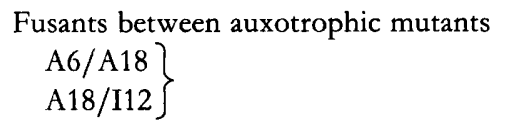 & Protoplast fusion & $\begin{array}{l}\text { Colonies like those of IV but thicker in } \\
\text { appearance and with more aerial mycelia }\end{array}$ \\
\hline VII & $\begin{array}{l}\text { Mitotic sectors of intergeneric } \\
\text { hybrids }\end{array}$ & & $\begin{array}{l}\text { In general, thin, filmy white colonies like } \\
\text { those of category IV; occasionally, sectors } \\
\text { with a distinctly slow growth rate } \\
\text { One sector, S6, obtained from A6/21501-4B } \\
\text { appeared wrinkled and non-hairy had less } \\
\text { aerial hyphae than the other sectors from the } \\
\text { same hybrid }\end{array}$ \\
\hline VIII & $\begin{array}{l}\text { Mitotic sectors of fusants } \\
\text { Y. lipolytica } 1529 \text { A bis } 1 \\
\text { Y. lipolytica } 21501-4 \mathrm{~B} \text { lys } 5 \text { leu } 2 \\
\text { ade1 xpr2 } \\
\text { Y. lipolytica A ura } 3 \text { leu2 lys } 11\end{array}$ & & $\begin{array}{l}\text { In general, thin, filmy white colonies like } \\
\text { those of category IV; occasionally, sectors } \\
\text { with a distinctly slow growth rate }\end{array}$ \\
\hline
\end{tabular}

produce wine from starchy substrates (Merican \& Yeoh, 1989).

Very little is known about the genetics of this yeast and, as far as we are aware, only two papers which reported studies of intergeneric hybrids between Saccharomycopsis and Yarrowia have previously been published (Nga et al., 1992, 1994). We are particularly interested in developing a system for genetic analysis in S. fibuligera which requires a haploid strain. For this purpose an intergeneric hybrid between $S$. fibuligera 193 met and a strain of the closely related dimorphic yeast, $Y$. lipolytica 1529 A his1 was produced by mass mating. The intergeneric hybrid was extremely unstable in prolonged vegetative culture on YPSS and produced numerous mitotic segregants. A large number of the mitotic segregants were mitotically unstable. Among the mitotic segregants, however, there was a mitotically stable, putative haploid strain, N14i60 met (Nga et al., 1994). By UV mutagenesis of this strain, auxotrophic mutants were readily obtained. Diploid fusants were obtained between pairs of these mutants by protoplast fusion. When single colonies of the fusants were grown on YPSS agar for $14 \mathrm{~d}$ at $28^{\circ} \mathrm{C}$ mitotic sectors, some of which were stable and haploid, were obtained. By systematic analysis of the phenotypic classes of the haploid mitotic sectors of the fusants, linkage relationships between the genetic markers of the parent strains were determined (Nga et al., 1994). As we now have a pedigree of $S$. fibuligera strains in our stock, we set out to determine if these strains could be classified by differences in their electrophoretic karyotypes. We also studied the pattern of hybridization signals for specific genes of $S$. fibuligera for the intergeneric hybrid, A6/21501-4B, and its mitotic segregants.

\section{METHODS}

Strains of yeast. The origin and characteristics of the strains of S. fibuligera (8014 met, 193 met, 2610, 56 met, N14i60 met, A6, A18 and I12) and of $Y$. lipolytica (1529 A bis1, 21501-4B leu2 lys5 ade 1 $x p r 2$ and $A$ ura 3 leu2 lys 11 ) studied are given in Table 1 . The interrelationships of the yeast strains of direct significance to this study are presented in the pedigree chart in Fig. 1. 


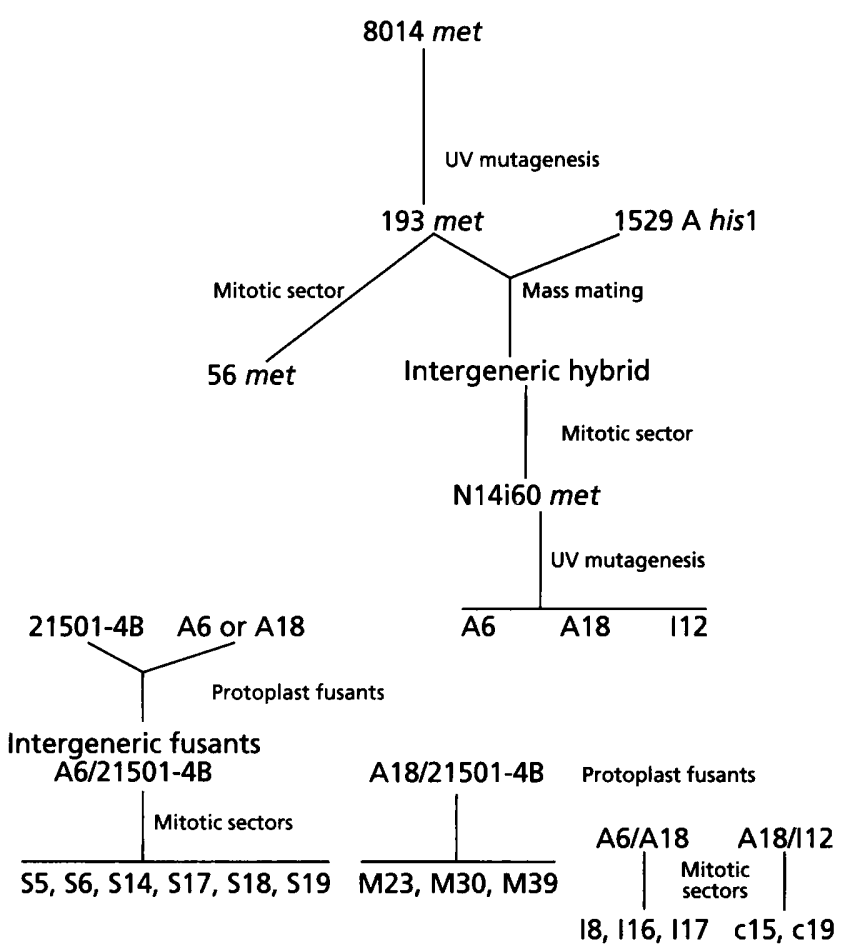

Fig. 1. Pedigree of yeast strains used in this study.

Saccharomyces cerevisiae SH964 and Schizosaccharomyces pombe L975 were from the stock of Y. Oshima, Department of Biotechnology, Faculty of Engineering, Osaka University, Japan. They were used to provide the standard size markers for the pulsed-field gel electrophoresis (PFGE) runs.

Media. The media for the growth of the strains used in these studies were those of Nga et al. (1992). The recipes for complex yeast extract/peptone/dextrose medium (YEPD), and Difco Yeast Nitrogen Base (without amino acids) (YNB) minimal medium were described by Nga et al. (1992). Supplementation of YNB when required (for classification) was as follows: $1.5 \%$ $(\mathrm{w} / \mathrm{v})$ starch, $2 \%(\mathrm{w} / \mathrm{v})$ glucose, $1 \%(\mathrm{v} / \mathrm{v})$ tributyrin, $20 \mathrm{mg} \mathrm{l}^{-1}$ each of adenine, arginine and methionine, and $30 \mathrm{mg} \mathrm{l}^{-1}$ each of leucine and lysine. The recipes of YPSS and yeast extract agar (YEA) were described by Nga et al. (1992).

Protoplast fusion. Preparation of protoplasts of yeast strains and protoplast fusion between pairs of yeast strains were by the techniques of $\mathrm{Nga}$ et al. (1992). Following the treatment of the protoplasts of the two strains with $4 \mathrm{ml} 25 \%(\mathrm{v} / \mathrm{v})$ PEG in osmotic stabilizing buffer (OSB) and incubation at $30^{\circ} \mathrm{C}$ for $30 \mathrm{~min}$, an equal volume of OSB was added to the suspension, followed by centrifugation. The pellet was washed once in $2 \mathrm{ml}$ OSB and resuspended in $2 \mathrm{ml} O S B$. Aliquots from this preparation, and diluted portions thereof, were dispensed onto $3.5 \mathrm{ml}$ molten YNBG agar containing $1 \mathrm{M}$ sorbitol (YNBGS) and overlaid onto YNBGS agar plates. Depending on the specific crosses, the YNBGS in the overlay was appropriately supplemented with lysine, adenine or methionine.

Mitotic sectoring of fusants. S. fibuligera 193 met/Y. lipolytica 1529 A bis 1 intergeneric hybrid, and the protoplast fusants between pairs of auxotrophic mutant strains derived from N14i60 met, were inoculated individually onto the centre of YPSS plates and incubated at $28^{\circ} \mathrm{C}$ for $14 \mathrm{~d}$. These strains could be distinguished from the haploid strains as the latter were stable and did not give sectors in mitotic sectoring tests.
Mass mating. Crossing between S. fibuligera 193 met and $Y$. lipolytica 1529 A bis1 was by the method of Gaillardin et al. (1973). Intergeneric hybrids were obtained as described by $\mathrm{Nga}$ et al. (1992).

PFGE. The protocol for preparing DNA of yeast strains to be separated by PFGE was that of De Jonge et al. (1986). Resolution of the yeast chromosomal DNA was carried out by using the LKB-Pharmacia Pulsaphor apparatus equipped with a hexagonal electrode array (Koh, 1993; Yip, 1993). The running conditions of the Pulsaphor consisted of three sequential separating steps as follows: $80 \mathrm{~V}$ with a $1200 \mathrm{~s}$ pulse time for $48 \mathrm{~h} ; 50 \mathrm{~V}$ with a $1800 \mathrm{~s}$ pulse time for $48 \mathrm{~h}$; and then $50 \mathrm{~V}$ with a $2700 \mathrm{~s}$ pulse time for $48 \mathrm{~h}$. Electrophoresis was carried out in $1 \%(\mathrm{w} / \mathrm{v})$ agarose gel with $0.5 \times$ Tris/acetate/EDTA cooling buffer at $14^{\circ} \mathrm{C}$.

Labelling with isotope, hybridization probe and preparation of autoradiograms. Synthesis of uniformly labelled probes was with $\left[\alpha-{ }^{32} \mathrm{P}\right] \mathrm{dC}$ TP by the random priming method. Southern hybridizations of nylon membrane impregnated with DNA and preparation of autoradiograms with Kodak XAR-5 X-ray film were carried out by standard methods (Sambrook $e t$ al., 1989). The $\alpha$-amylase ( $A L P 1)$ gene probe was prepared from the $1.88 \mathrm{~kb}$ XboI-StuI fragment of the plasmid pSfALP1 of Yamashita et al. (1985a). The glucoamylase (GLU1) gene probe was the $2.54 \mathrm{~kb}$ PstI fragment of the plasmid pSfGLU ES1 of Yamashita et al. (1985b). The rDNA gene probe was the $7 \cdot 7 \mathrm{~kb}$ HindIII fragment of plasmid pINA 24 obtained from van Heerikhuizen et al. (1985).

\section{RESULTS AND DISCUSSION}

\section{Characteristics of the intergeneric hybrids and protoplast fusants between auxotrophic mutants}

In the case of the intergeneric hybrid, A6/21501-4B, the fusant was isolated on YNB supplemented with glucose and lysine (Chiu, 1992). The hybrid grew only when YNBG was supplemented with lysine. With the intergeneric hybrid A18/21501-4B, as expected, the fusant was able to grow on YNBG supplemented with adenine. This result could be explained on the basis that in both the parent strains, lesions had occurred in the gene for the same enzyme in the biosynthetic pathway of adenine. Protoplast fusants, A6/A18 and A18/I12, were able to grow on YNBG.

A distinctive characteristic of the intergeneric hybrids and the protoplast fusants between auxotrophic mutants was that they were mitotically unstable in vegetative culture on YPSS at $28^{\circ} \mathrm{C}$ for $14 \mathrm{~d}$.

Phenotypes of the intergeneric hybrid, A6/215014B, the protoplast fusants $A 6 / A 18$ and $A 18 / 112$, and some of their mitotic sectors

S. fibuligera produces glucoamylase and $\alpha$-amylase. $\mathrm{Glu}^{+}$ colonies produced an opaque halo on starch agar due to formation of turbid precipitates, whereas $\mathrm{Alp}^{+}$colonies produced a clear halo. Glucoamylase catalyses the release of glucose molecules from the non-reducing ends of starchy materials and other $\alpha$-glucopyranosides. $\alpha$ Amylase randomly hydrolyses $\alpha$-1,4-glucoside linkages of amylose and amylopectin, but not $\alpha-1,6$-glucoside link- 


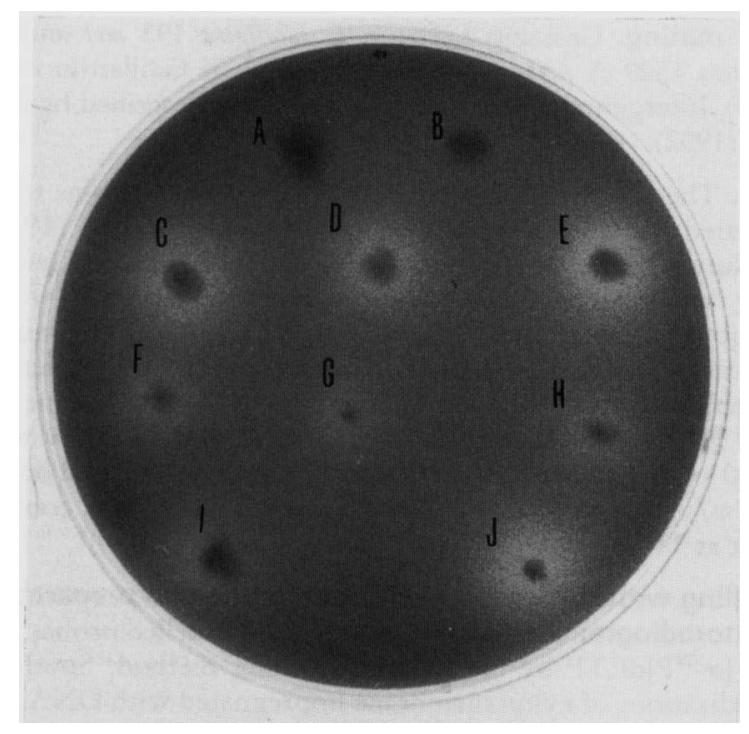

Fig. 2. Hydrolysis of starch of yeast strains on YPSS medium observed as haloes around the colonies. A, Y. lipolytica A his1; B, Y. lipolytica 21501-4B leu2 lys5 ade1 xpr2; C, S. fibuligera A6; $D$, S. fibuligera A18; E, S. fibuligera/Y. lipolytica intergeneric hybrid A6/21501-4B; F, S. fibuligera S5; G, S. fibuligera S6; H, S. fibuligera S14; I, S. fibuligera S17; J, S. fibuligera S19.

ages of amylopectin. For the colonies of A6 and A18, clear haloes were observed after growth on YPSS for $2 \mathrm{~d}$. Colonies of the intergeneric hybrid between $\mathrm{A} 6$ and $Y$. lipolytica 21501-4B also showed a clear halo around the colony on YPSS. On this medium, colonies of $Y$. lipolytica 21501-4B did not show a halo; this result was expected as $Y$. lipolytica is not amylolytic. Strains A6, A18 and A6/21501-4B showed a similar growth rate on YPSS. When cells of the hybrid, A6/21501-4B, were inoculated onto YPSS as single colonies and incubated for $14 \mathrm{~d}$ at $28^{\circ} \mathrm{C}$, mitotic sectors were observed. Some of the sectors were unstable and gave sectors when their cells were inoculated onto YPSS and tested for mitotic sectoring; other sectors from the hybrid were stable when tested for mitotic sectoring. Six stable mitotic segregants obtained from the intergeneric hybrid, A6/21501-4B, showed a slower growth rate than the parent hybrid. Colonies of one of the segregants, S6, showed a distinctly slower growth rate with less aerial hyphae when compared with the other five segregants, S5, S14, S17, S18 and S19 (Table 1; Yip, 1993). Furthermore, the surface of the colonies of S6 appeared wrinkled and non-hairy, i.e. similar to $Y$. lipolytica. However, colonies of S6 on YPSS could be easily distinguished from those of $Y$. lipolytica 21501-4B on the same medium. Colonies of the other five segregants had aerial hyphae, a non-wrinkled appearance and amylolytic activity, and thus resembled S. fibuligera A6.

All six mitotic segregants were considered to be putative haploids as they were mitotically stable and did not give sectors when inoculated onto YPSS. When five of the segregants were grown on YPSS as single colonies for $2 \mathrm{~d}$ at $28^{\circ} \mathrm{C}$, haloes were observed, but with sizes smaller than those of A6, A18 and A6/21501-4B (Fig. 2; Yip, 1993) with strain $\mathrm{S} 6$ giving a distinctly smaller halo than the others. Strains S5, S14 and S17 gave haloes which were smaller than S19 but larger than S6. A distinctive feature was that the four strains, S5, S6, S14 and S17, gave haloes which had a cloudy appearance in contrast to the bright or clear haloes of A6, A18, A6/21501-4B and S19. To explain this phenomenon more precisely, studies were made of the electrophoretic karyotypes of these strains and of Southern hybridization patterns with the probes for the $A L P 1$ and GLU1 genes (see below). These results are in agreement with the view that the haploid mitotic segregants arose from the diploid parent hybrid by a process of haploidization during which chromosomal assortment had occurred.

As for the protoplast fusants, A6/A18 and A18/I12, all the stable mitotic segregants obtained from them from mitotic sectoring had, in general, a thin, filmy white phenotype (Table 1). Colonies of the segregants on YPSS had aerial hyphae, were non-wrinkled and amylolytic and resembled $S$. fibuligera strains $\mathrm{A} 6$ and A18. Mitotic segregants with parental and non-parental phenotypes were obtained from these fusants (Chiu, 1992).

\section{Electrophoretic karyotypes of yeast strains}

The chromosomal DNA banding patterns of the yeast strains used in this study are represented by electrophoretic karyotypes in Fig. 3. In these studies, standard size markers for the chromosomes were provided by the DNA of Schiz. pombe and Sacc. cerevisiae. The results showed that four chromosomal banding patterns for the $S$. fibuligera strains could be distinguished as follows: I, 2610 and 8014 met, with seven bands; II, 193 met, with eight bands; III, 56 met, with six bands; and IV, N14i60 met and the auxotrophic mutants derived from it, the intergeneric hybrids A6/21501-4B and A18/21501-4B, the protoplast fusants between pairs of the auxotrophic mutants, and the mitotic segregants of the hybrids and fusants, with seven bands. The mobilities of the seven bands of these strains correspond with one another. $Y$. lipolytica 1529 A bis 1 had six bands and 21501-4B six bands. Chromosomal DNA bands 5 and 6 of $Y$. lipolytica 21501-4B are of sizes which corresponded with bands 6 and 7 of the type IV strains, respectively. As can be observed in Fig. 3, strain 8014 met differs from 193 met in that it lacks a band corresponding to band 6 of the latter. Bands 1-4 of strains 193 met and 8014 met correspond with one another. Band 2 of both these strains is larger than band 2 of the type IV strains. Band 6 of the type IV strains corresponds with band 7 of 193 met and band 6 of 8014 $m e t$, and the band 5 of the type IV strains corresponds with band 5 of 193 met, also being smaller than band 5 of 8014 met (Koh, 1993; Yip, 1993).

\section{Probing by Southern hybridization with $\alpha$-amylase (ALP1) and glucoamylase (GLU1) genes}

A comparison of the electrophoretic karyotypes of the mitotic segregants with their phenotypes revealed that although these strains were clearly distinguishable phenotypically, they could not be separated by their DNA 
(a)

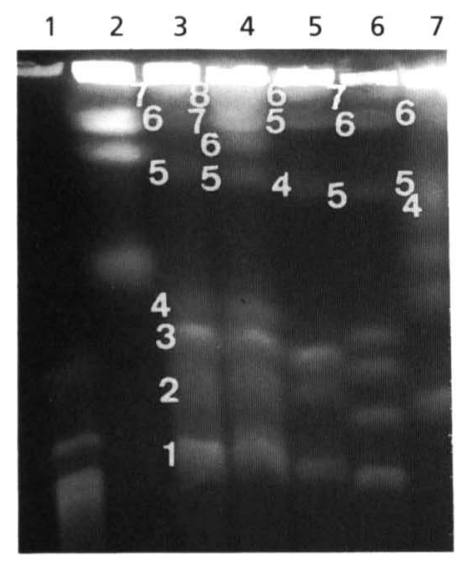

(b)

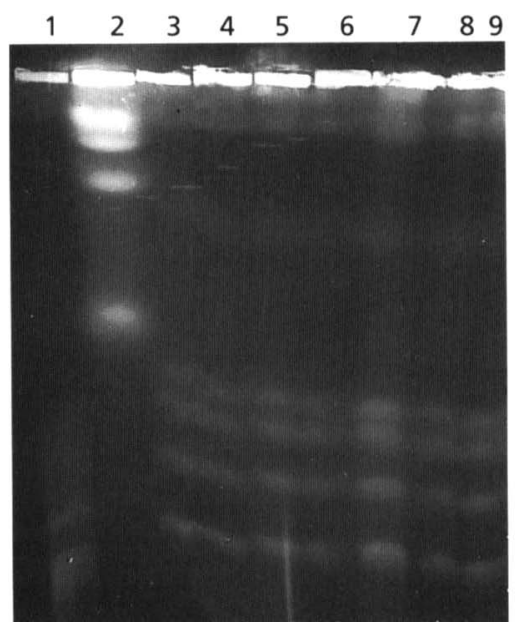

(c)

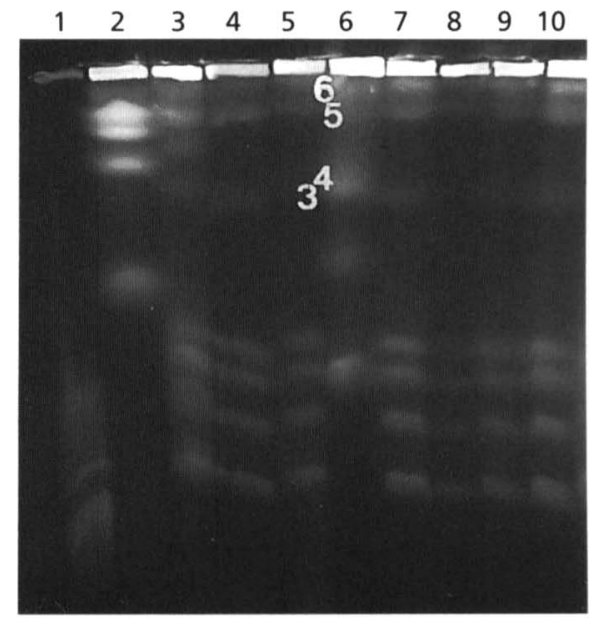

Fig. 3. PFGE karyotyping of yeast strains. (a) Lane 1, Sacc. cerevisiae; lane 2, Schiz. pombe; lane 3, S. fibuligera 8014 met; lane 4, S. fibuligera 193 met; lane 5, S. fibuligera 56 met; lane 6, S. fibuligera N14i60 met; lane 7, Y. lipolytica A his1. (b) Lane 1, Sacc. cerevisiae; lane 2, Schiz. pombe; lane 3, S. fibuligera A6; lane 4, S. fibuligera fusant A6/A18; lanes 5 and 6, mitotic sectors 116 and 117 of A6/A18; lane 7, S. fibuligera fusant A18/112; lane 8, S. fibuligera I12; lane 9, mitotic sector C15 of A18/I12. (c) Lane 1, Sacc. cerevisiae; lane 2, Schiz. pombe; lane 3, S. fibuligera 193 met; lane 4, S. fibuligera N14i60 met; lane 5, S. fibuligera A18; lane 6; Y. lipolytica 21501-4B leu2 lys5 ade1 xpr2; lane 7, S. fibuligera/Y. lipolytica intergeneric hybrid A18/21501-4B; lanes 8, 9 and 10, mitotic sectors M23, M30 and M39 of A18/21501-4B.

banding patterns in PFGE studies. This agrees with the results of a recent study by Rustchenko-Bulgac \& Howard (1993) in which the phenotypic variability of a number of characters in strains of Candida albicans did not correspond with the specific patterns of chromosomal alterations in PFGE studies.

In the study on Southern hybridization, the DNA bands of the intergeneric hybrid A6/21501-4B and the four mitotic segregants, S5, S6, S14 and S17, were probed with $A L P 1$ and GLU1 (Yip, 1993). In the case of ALP1, hybridization signals were observed for DNA band 1 for strains A6, A6/21501-4B, and the four segregants, but not for Y. lipolytica 21501-4B (Fig. 4). The reduced amylolytic activity of strains S5, S6, S14 and S17 as compared with A6 was therefore not due to the absence of the ALP1 gene in chromosome 1 . With the GLU1 probe hybridization signals were observed for bands 6 and 7 for $A 6$ and A6/21501-4B, indicating that their chromosomal bands 6 and 7 were similar to those of strain A6, whereas in the cases of the segregants S6 and S14 signals were only observed for band 7 (Fig. 5). In the cases of S5 and S17, hybridization signals were observed only for band 6 . No hybridization signals were observed for the DNA bands of $Y$. lipolytica 21501-4B. These results suggest that chromosomal assortment occurred at mitosis, giving rise to haploid strains with different haploid complements of chromosomes.

Taking all the results into account, three classes of mitotic segregants of the intergeneric hybrid, A6/21501-4B, can be distinguished. As all of the four strains, S5, S6, S14 and S17, have seven chromosomal DNA bands with corresponding mobilities, the differences between these strains cannot be due to aneuploidy. With the GLU1 probe, strains S5 and S17 gave hybridization signals with chromosomal band 6, whereas strains S6 and S14 hybridized with chromosomal band 7 . In the cases of $\mathrm{S} 5$ and S17, chromosomal band 7 of A6 could have been substituted by chromosome 6 of Y. lipolytica 21501-4B, whereas the S6 chromosome 6 of A 6 could have been replaced by chromosome 5 of $Y$. lipolytica 21501-4B. This result suggests that the GLU1 gene on chromosomes 6 or 7 may be absent in these segregants with no distinct undesirable or lethal consequence to the strain. Strain S14 is however different from S6 in that S14 has hairy aerial hyphae. Of particular significance was the finding that a few spontaneous segregants were obtained from S14 in mitosis which were strongly amylolytic. No such segregants were obtained from S6. It seems probable from these results that S14 might contain a specific chromosomal rearrangement in its chromosome 6 so that no clear hybridization signals were observed for the GLU1 probe. Furthermore, strain S6, which has a low amylolytic activity, is characterized by its wrinkled and non-hairy appearance. A plausible explanation for this is that its genome contains a chromosome of $Y$. lipolytica 21501-4B which carries a gene required for the expression of this phenotype. By the same reasoning it can be concluded that this $Y$. lipolytica gene or chromosome is not present in S5, S14 or S17. The substitution of chromosomal band 7 of A6 by band 6 of Y. lipolytica 21501-4 in S5 and S17 did not bring about such a clear change in morphological characteristics.

When the rDNA probe of $Y$. lipolytica was used to detect differences among strains $A 6, S 5$ and $S 6$, it was found that while A6 gave signals of hybridization for chromosomal bands 6 and 7, in the case of S5 and S6 signals were observed for chromosomal bands 6 (not shown) and 7, 
(a)

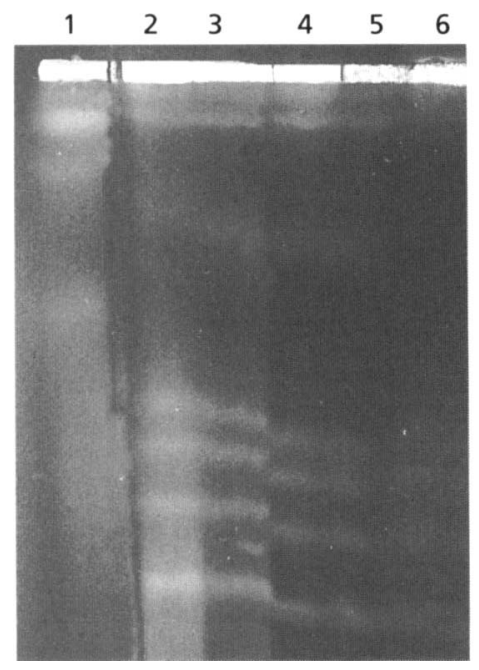

(c)

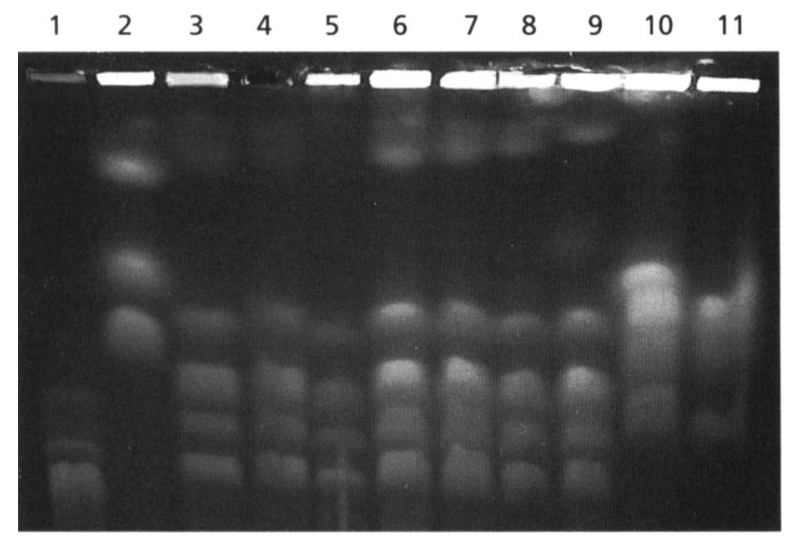

(d)

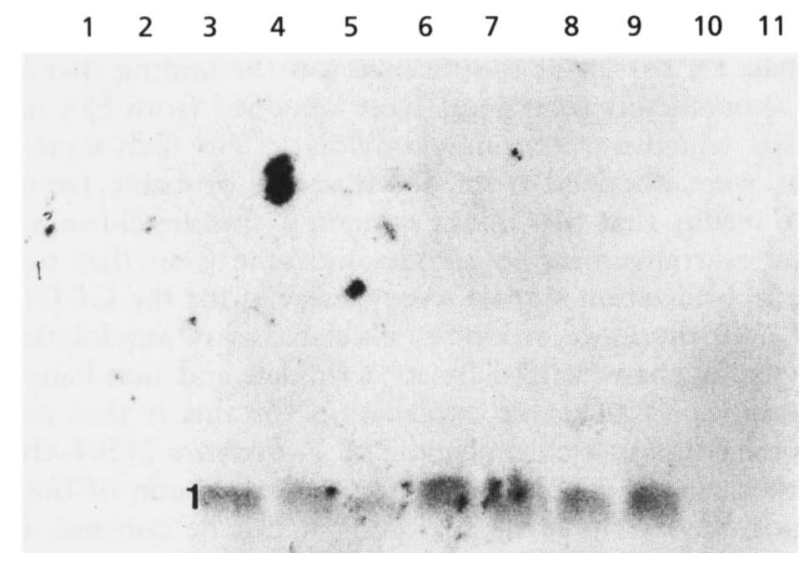

Fig. 4. Hybridization of the $S$. fibuligera $A L P 1$ gene probe $(b, d)$ to a blot of the PFGE $(a, c)$ panel of yeast strains. $(a, b)$ Lane 1, Schiz. pombe; lane 2, S. fibuligera/Y. lipolytica intergeneric hybrid $A 6 / 21501-4 B$; lane 3 , S. fibuligera $A 6$; lane 4, S. fibuligera S5; lane 5, S. fibuligera S6; lane 6, S. fibuligera S14. (c, d) Lane 1, Sacc. cerevisiae; lane 2, Schiz. pombe; lane 3, S. fibuligera N14i60; lane 4, S. fibuligera A2.1; lane 5, S. fibuligera A18; lane 6, S. fibuligera fusant A18/A2.1; lanes 7, 8 and 9, mitotic sectors 5, 16 and 25 of A18/A2.1; lane 10, Y. lipolytica A ura3 leu2 lys11; lane 11, Y. lipolytica 1529 A his1. (a)

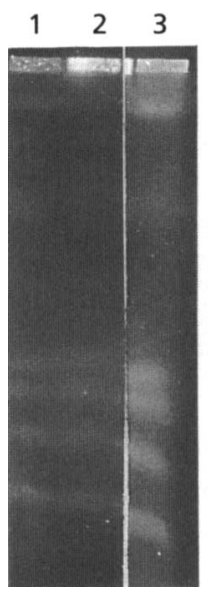

(b)

Fig. 5. Hybridization of the $S$. fibuligera GLU1 gene probe (b) to a blot of the PFGE (a) panel of yeast strains. Lane 1, $S$. fibuligera S5; lane 2, S. fibuligera S6; lane 3, S. fibuligera/Y. lipolytica intergeneric hybrid A6/21501-4B.

(a)

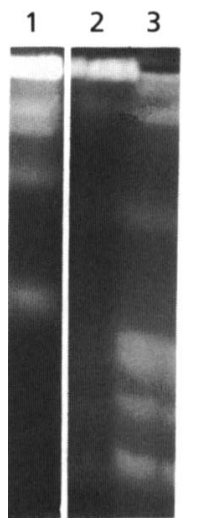

(b)

123

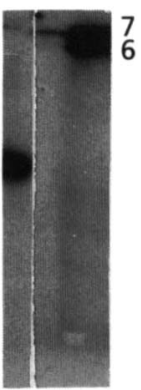

Fig. 6. Hybridization of the $Y$. lipolytica rRNA genes $(b)$ to a blot of the PFGE (a) panel of yeast strains. Lane 1, Schiz. pombe; lane 2, S. fibuligera S6; lane 3, S. fibuligera A6.

respectively (Fig. 6). Previous studies (Yip, 1993) showed that, using the same rDNA probe, $Y$. lipolytica 21501-4B gave signals of hybridization for chromosomal DNA bands 1 and 4 . These results suggest that the rDNA genes of $S$. fibuligera strains are located on chromosomes 6 and 7. Similar results concerning the chromosomal location of the GLU1 gene were obtained with the GLU1 probe. We take this correlation in the patterns of hybridization for the probes GLU1 and rDNA for strains S5 and S6 to be more definitive evidence in support of our finding that chromosome 7 of S5 and S17 was indeed chromosome 6 of $Y$. lipolytica 21501-4B, and that chromosome 6 of S6 was chromosome 5 of 21501-4B. 
Thus, by a combined analysis of the karyotypes of genetically different strains of $S$. fibuligera, with a study of phenotypic differences of intergeneric hybrids and their mitotic segregants and the patterns of hybridization signals for specific genes, a more discriminative means of assessing the phenotypic differences of the yeast strains studied was possible. Of special interest is the possibility of using the weakly amylolytic strain, S5, as a recipient in transformation experiments which employ plasmids carrying the glucoamylase gene, GLU1, and the $26 \mathrm{~S}$ ribosomal DNA sequence of $S$. fibuligera. Preliminary results indicate that integration of the linearized $8.94 \mathrm{~kb}$ plasmid, pYLN1826GLU1, into the corresponding rDNA locus of S5, produces strongly amylolytic transformants.

\section{ACKNOWLEDGEMENTS}

This work was supported by National University of Singapore Grant RP 910417. We thank Miss Siti M. Masnor for assistance in the preparation of this manuscript.

\section{REFERENCES}

Barnett, J. A., Payne, R. W. \& Yarrow, D. (1983). Yeasts: Characteristics and Identification. Cambridge: Cambridge University Press.

Chiu, L. L. (1992). The development of a genetic analysis system for Saccharomycopsis fibuligera-Yarrowia lipolytica recombinants. MSc thesis, National University of Singapore, Singapore.

De Jonge, P., De Jonge, C. M., Meijers, R., De Steensma, H. Y. \& Scheffers, W. A. (1986). Orthogonal-field-alternation gel electrophoresis banding patterns of DNA from yeasts. Yeast 2, 193-204.

Gaillardin, C. M., Charoy, V. \& Heslot, H. (1973). A sudy of copulation, sporulation and meiotic segregation in Candida lipolytica. Arch Microbiol 92, 69-83.

van Heerikhuizen, H., Ykema, A., Klootwijk, J., Gaillardin, C., Ballas, C. \& Fournier, P. (1985). Heterogeneity in the ribosomal RNA genes of the yeast Yarrowia lipolytica: cloning and analysis of two size classes of repeats. Gene 39, 213-222.
Koh, S. I. (1993). Genetic studies of Saccharomycopsis fibuligera and Yarrowia lipolytica. MSc thesis, National University of Singapore, Singapore.

Kreger-van Rij, N. J. W. (1984). The Yeasts: a Taxonomic Study, 3rd edn. Amsterdam: Elsevier.

Lodder, J. (1970). The Yeasts: a Taxonomic Study, 2nd edn. Amsterdam: North Holland.

Machida, M., Ohtsuki, I., Fukui, S. \& Yamashita, I. (1988). Nucleotide sequences of Saccharomycopsis fibuligera genes for extracellular $\beta$-glucosidase as expressed in Saccharomyces cerevisiae. Appl Environ Microbiol 54, 3147-3155.

Merican, Z. \& Yeoh, Q. L. (1989). Tapai processing in Malaysia: a technology in transition. In Industrialisation of Indigenous Fermented Foods, pp. 169-189. Edited by K. H. Steinkraus. New York : Marcel Dekker.

Nga, B. H., Abu Bakar, F. D., Loh, G. H., Chiu, L. L., Harashima, S., Oshima, Y. \& Heslot, H. (1992). Intergeneric hybrids between Saccharomycopsis fibuligera and Yarrowia lipolytica. J Gen Microbiol 138, 223-227.

Nga, B. H., Chiu, L. L., Koh, S. I., Yip, C. W., Harashima, S. \& Oshima, Y. (1994). Occurrence of genetic segregation in a putative haploid strain of Endomyces fibuliger met by spontaneous sectoring of protoplast fusants. World J Microbiol \& Biotechnol 10, 465-471.

Rustchenko-Bulgac, E. P. \& Howard, D. H. (1993). Multiple chromosomal and phenotypic changes in spontaneous mutants of Candida albicans. J Gen Microbiol 139, 1195-1207.

Sambrook, J., Fritsch, E. F. \& Maniatis, T. (1989). Molecular Cloning: a Laboratory Manual, 2nd edn. Cold Spring Harbor, NY: Cold Spring Harbor Laboratory.

Yamashita, I., Itoh, T. \& Fukui, S. (1985a). Cloning and expression of the Saccharomycopsis fibuligera $\alpha$-amylase gene in Saccharomyces cerevisiae. Agric Biol Chem 49, 3089-3091.

Yamashita, I., Itoh, T. \& Fukui, S. (1985b). Cloning and expression of Saccharomycopsis fibuligera glucoamylase gene in Saccharomyces cerevisiae. Appl Microbiol Biotechnol 23, 130-133.

Yip, C. W. (1993). Studies of electrophoretic karyotypes and assignment of DNA probes in Saccharomycopsis fibuligera and Yarrowia lipolytica. BSc thesis, National University of Singapore, Singapore.

Received 6 June 1994; revised 21 September 1994; accepted 14 November 1994. 